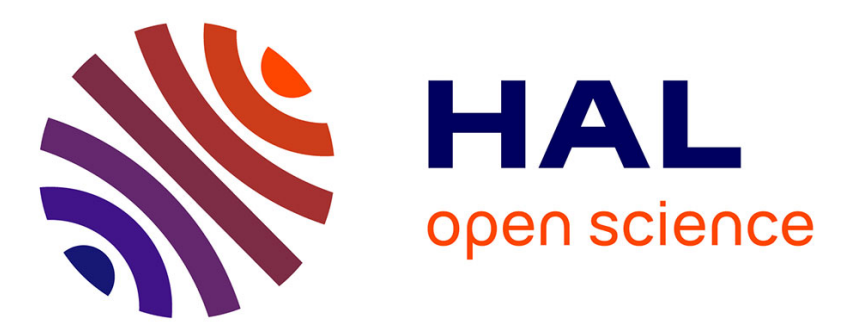

\title{
The Sudden-Polarization Effect and its Role in the Ultrafast Photochemistry of Ethene
}

\author{
Alexandra Viel, Robert P. Krawczyk, Uwe Manthe, Wolfgang Domcke
}

\section{To cite this version:}

Alexandra Viel, Robert P. Krawczyk, Uwe Manthe, Wolfgang Domcke. The Sudden-Polarization Effect and its Role in the Ultrafast Photochemistry of Ethene. Angewandte Chemie International Edition, 2003, 42 (29), pp.3434-3436. 10.1002/anie.200351193 . hal-01118363

\section{HAL Id: hal-01118363 https://hal.science/hal-01118363}

Submitted on 3 Jun 2020

HAL is a multi-disciplinary open access archive for the deposit and dissemination of scientific research documents, whether they are published or not. The documents may come from teaching and research institutions in France or abroad, or from public or private research centers.
L'archive ouverte pluridisciplinaire HAL, est destinée au dépôt et à la diffusion de documents scientifiques de niveau recherche, publiés ou non, émanant des établissements d'enseignement et de recherche français ou étrangers, des laboratoires publics ou privés. 


\title{
The Sudden-Polarization Effect and its Role in
}

\section{the Ultrafast Photochemistry of Ethene ${ }^{* *}$}

\author{
Alexandra Viel, Robert Krawczyk, Uwe Manthe and Wolfgang Domcke* \\ Institute of Physical and Theoretical Chemistry, \\ Technical University of Munich \\ D-85747 Garching, Germany \\ Fax: +898928913622 WOLFGANG.DOMCKE@CH.TUM.DE
}

February 14, 2003

The term sudden polarization, describing the charge separation in excited singlet states of twisted polyenes, especially of ethene, has been introduced in the mid 70 s by Salem. ${ }^{1}$ The ionic character and the high polarization upon a small perturbation of these valence excited states has been pointed out as early as in 1971 by Wulfman and $\mathrm{Kumei}^{2}$ for alkenes. Bonačić-Koutecký et al. ${ }^{3}$ were the first to perform an ab initio study of the charge separation in $90^{\circ}$-twisted ethene as a function of an internal perturbation: the one-sided pyramidalization of a $\mathrm{CH}_{2}$ group. To our knowledge, all following studies of the suddenpolarization effect of ethene have considered only one-sided pyramidalization as internal geometrical distortion to break the $D_{2 d}$ symmetry. ${ }^{4} 8$ We discuss here

${ }^{* *}$ A.V. acknowledges financial support via a Marie Curie fellowship of the European Community program Improving Human Research Potential and the Socio-economic Knowledge Base under the contract number HPMFCT-2000-00840. U. M. and W. D. acknowledge financial support by the Deutsche Forschungsgemeinschaft. 
the effects of one-sided scissoring as another internal perturbation and show that the transfer of electronic charge between the $\mathrm{CH}_{2}$ groups occurs in first order of this perturbation, whereas the effect is of second order in the pyramidalization. Moreover, we show that the one-sided scissors mode plays an essential role in the nonadiabatic photochemistry of ethene.

The sudden-polarization effect can be understood as the direct consequence of a small dissymmetry of the molecule. At the ground-state equilibrium geometry, ethene is planar and belongs to the symmetry group $D_{2 h}$. In this group, the delocalized molecular orbitals, $\pi$ and $\pi^{*}$, span the $b_{3 u}$ and $b_{2 g}$ representations, respectively (within Mulliken's axis definition ${ }^{9}$ ). The three relevant valence states $^{9} \mathrm{~N}\left(\pi^{2}\right), \mathrm{V}\left(\pi \pi^{*}\right)$ and $\mathrm{Z}\left(\pi^{* 2}\right)$ thus have $\mathrm{A}_{g}, \mathrm{~B}_{1 u}$ and $\mathrm{A}_{g}$ symmetry. Upon torsion of the $\mathrm{CH}_{2}$ groups around the $\mathrm{CC}$ bond, the symmetry is lowered to $D_{2}$. In this symmetry group, the $\pi\left(\pi^{*}\right)$ orbital spans the $b_{3}\left(b_{2}\right)$ representation. This holds for all torsional angles $\varphi$ not equal to $0^{\circ}$ (planar geometry) and $90^{\circ}$ (perpendicular geometry). For $\varphi=90^{\circ}$, the symmetry group is $D_{2 d}$. In this group, both $\pi$ and $\pi^{*}$ belong to the $e$ representation. At this particular geometry, any linear combination of the $\pi$ and $\pi^{*}$ orbitals is acceptable, in particular the orbitals $\phi_{r}$ and $\phi_{l}$ which are localized on the right and left $\mathrm{CH}_{2}$ groups, respectively. Since the two $\mathrm{CH}_{2}$ groups are rigorously symmetry equivalent, the molecular orbitals have to reproduce a zero dipole moment. The symmetrized linear combinations $\phi_{r}^{2}-\phi_{l}^{2}$ and $\phi_{r}^{2}+\phi_{l}^{2}$, which have $\mathrm{B}_{2}$ and $\mathrm{A}_{1}$ symmetry, respectively, are acceptable solutions. They correspond to two excited states, V and $\mathrm{Z}$, which do not have to be degenerate by symmetry. They are nevertheless close in energy, with the $\mathrm{Z}$ state being approximately $0.1 \mathrm{eV}$ below the $\mathrm{V}$ state. ${ }^{6}$ The $\mathrm{V}$ and $\mathrm{Z}$ potential-energy functions cross at $\varphi \simeq 82^{\circ}, 6,10$ since the $\mathrm{Z}$ state is much higher in energy than the V state for planar geometry. If, for some reason, one side of the molecule is distinguished, an asymmetry of the electronic wave 
functions results, leading to charge separation. ${ }^{11}$ Because of the small overlap between the left and right orbitals and the small energy difference between the $\mathrm{V}$ and $\mathrm{Z}$ states of perpendicular ethene, the localization of the charge is pronounced, even for small perturbations. Both one-sided pyramidalization as well as one-sided scissoring lower the $D_{2 d}$ symmetry of perpendicular ethene to $C_{s}$ and $C_{2 v}$, respectively, breaking the left-right symmetry and thus allowing for a non-zero dipole moment.

Due to the complexity of the electronic structure of the excited-states of ethene (due to pronounced valence-Rydberg mixing and strong multi-reference character of the electronic wave functions ${ }^{12} 15$ ), the $a b$ initio determination of the energies and dipole moments of the three lowest valence states is not an easy task and has been the subject of many publications. ${ }^{3,5} 7,11$ Figure 1 shows the dipole moment $\mu_{z}$ of the $\mathrm{V}$ and $\mathrm{Z}$ states obtained at the CASSCF level with a cc-pVTZ basis $\operatorname{set}^{16}$ as a function of one-sided pyramidalization $\vartheta$ (Figure 1a) and one-sided scissoring $\alpha$ (Figure 1b) for perpendicular ethene. Since upward and downward pyramidalizations are symmetry-equivalent, it is obvious that any physical observable is an even function of $\vartheta$. Consequently, for small $\vartheta$, the dipole moments depend not linearly, but quadratically, on $\vartheta$. This can be seen in Figure 1a, where the $\mu_{z}(\vartheta)$ functions exhibit a typical parabolic shape for small values of $\vartheta$. For the scissors angle, on the other hand, increases and decreases, respectively, from the ground-state equilibrium value are not symmetry-equivalent. Consequently, the dipole moments as a function of the scissors angle (Figure 1b), are linear in $\alpha$ close to the origin. For both curves, the beginning of saturation, visible by the change of curvature, occurs at around $\mu_{z}=1 \mathrm{au}$, corresponding to $\vartheta \simeq 20^{\circ}$ and $\alpha-\alpha_{0} \simeq 10^{\circ}$, respectively, where $\alpha_{0}=117.3^{\circ}$ is the $\mathrm{HCH}$ angle in the ground state of ethene.

Because of the linear dependence of $\mu_{z}$ on $\alpha$, versus a quadratic dependence 
of $\mu_{z}$ on $\vartheta$, the polarization induced by one-sided scissoring is more pronounced than the one induced by one-sided pyramidalization for small distortions. The slope of $\mu_{z}(\alpha)$ is even larger for $\varphi \simeq 82^{\circ}$, as one expects from the smaller energy difference between the $\mathrm{V}$ and $\mathrm{Z}$ states at this torsional angle. The magnitude of the dipole moment in the saturation region is very similar for both distortions $\left(\mu=1.4\right.$ au for $\vartheta=60^{\circ}, \mu=1.2$ au for $\left.\alpha-\alpha_{0}=30^{\circ}\right)$.

The sudden-polarization effect implies a pronounced modification in the electronic wavefunctions by a small perturbation of the symmetry. This rapid change of the electronic structure in response to small nuclear deformations implies a strong non-adiabatic coupling between the two excited states for nearperpendicular geometries. ${ }^{17}$ The effect of this coupling on the nonradiative decay of the $\mathrm{V}$ state has been investigated by two-dimensional wave-packet propagations of the photoinduced nuclear motion on the non-adiabatically coupled potential-energy surfaces of the three valence-type singlet states. A diabatic representation of the $\mathrm{N}, \mathrm{V}$ and $\mathrm{Z}$ states, including 6 of the 12 vibrational degrees of freedom, has been developed recently. ${ }^{18}$ In this model, based on accurate CASPT2 ab initio data, the diabatic states are coupled linearly by the antissymetric scissors mode and quadratically by the pyramidalization. A more detailed description of the six-dimensional potential-energy surfaces can been found in Ref 18 . To illustrate the impact of the sudden-polarization effect on the photochemical dynamics, two calculations have been performed, including either the one sided-scissoring or the one-sided pyramidalization together with the torsion. All other coordinates have been kept frozen at their ground-state equilibrium values. Figure 2 shows the time evolution, on a time scale of $50 \mathrm{fs}$, of the population probability of the initially $(t=0)$ prepared diabatic $\pi \pi^{*}$ state, which is, in $D_{2}$ symmetry, the (adiabatic) $V$ state. After vertical electronic excitation from the ground state to the $V$ state, the population probability stays 
close to unity during the first 7 fs. After this period, the population decreases in both calculations, but the drop of the population is nearly twice as large in the $\varphi, \alpha$ calculation than in the $\varphi, \vartheta$ calculation. The initial $7 \mathrm{fs}$ correspond to the time which the torsional wave-packet, initially located at the planar geometry, needs to reach the perpendicular geometry and thus the region of strong non-adiabatic coupling between the $\mathrm{V}$ and $\mathrm{Z}$ states. The coupling in the $\varphi, \alpha$ case is seen to be more efficient than in the $\varphi, \vartheta$ case, reflecting the fact that the vibronic coupling is linear in the former case, but quadratic in the latter case.

To conclude, we have shown that the sudden polarization in perpendicular ethene occurs in first order in the one-sided scissors deformation, and in second order in the one-sided pyramidalization. Although it has been emphasized in the literature that any geometrical distortion breaking the $D_{2 d}$ symmetry of perpendicular ethene will produce a charge separation, the distinguished role of the one-sided scissoring has not been recognized. Following the initial work of Bonačić-Koutecký et al., ${ }^{3}$ all subsequent researchers have focussed on the pyramidalization. The relevance of the first-order V-Z vibronic coupling by the scissors coordinate for the photoinduced dynamics has been demonstrated by quantum wave-packet calculations. These calculations reveal that the linear $\mathrm{V}-\mathrm{Z}$ coupling via the scissors mode provides a substantially faster flow of the electronic population probability out of the initially prepared V state than the quadratic coupling via the pyramidalization mode.

\section{References}

[1] L. Salem, Science 1976, 191, 822.

[2] C. E. Wulfman, S. Kumei, Science 1971, 172, 1061. 
[3] V. Bonačić-Koutecký, P. Bruckmann, P. Hiberty, J. Koutecký, C. Leforestier, L. Salem, Angew. Chem. Int. Ed. 1975, 14, 575.

[4] G. Berthier, B. Levy, L. Praud, Gazz. Chim. Ital. 1978, 108, 377.

[5] B. R. Brooks, H. F. Schaefer III, J. Am. Chem. Soc. 1979, 101, 307.

[6] R. J. Buenker, V. Bonačić-Koutecký, L. Pogliani, J. Chem. Phys. 1980, 73, 1836.

[7] I. D. Petsalakis, G. Theodorakopoulos, C. A. Nicolaides, R. J. Buenker, S. D. Peyerimhoff, J. Chem. Phys. 1984, 81, 3161.

[8] S. El-Taher, R. H. Hilal, T. A. Albright, Int. J. Quant. Chem. 2001, 82, 245.

[9] A. J. Merer, R. S. Mulliken, Chem. Rev. 1969, 69, 639.

[10] I. Ohmine, J. Chem. Phys. 1985, 83, 2348.

[11] L. Salem, Acc. Chem. Res. 1979, 12, 87.

[12] M. Ben-Nun, T. J. Martínez, Chem. Phys. 2000, 259, 237.

[13] V. Molina, M. Merchán, B. O. Roos, P.-A. Malmqvist, Phys. Chem. Chem. Phys. 2000, 2, 2211.

[14] S. Krebs, R. J. Buenker, J. Chem. Phys. 1997, 106, 7208.

[15] T. Müller, M. Dallos, H. Lischka, J. Chem. Phys. 1999, 110, 7176.

[16] T. H. Dunning, J. Chem. Phys. 1989, 90, 1007.

[17] G. Orlandi, P. Palmieri, G. Poggi, Chem. Phys. Letters 1979, 68, 251.

[18] R. P. Krawczyk, A. Viel, U. Manthe, W. Domcke, submitted to J. Chem. Phys. 2003. 

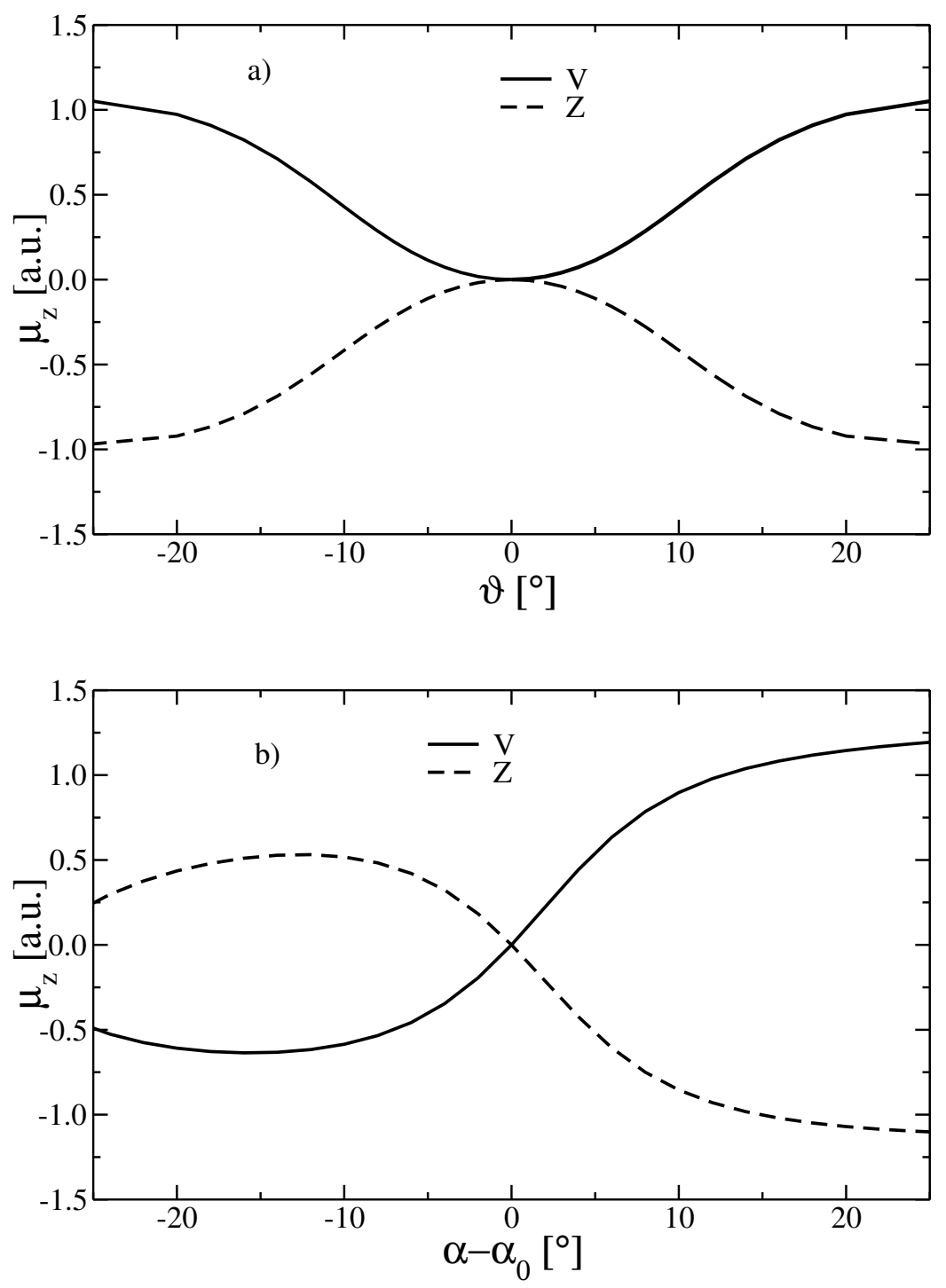

Figure 1: Dipole moment (in atomic units) along the $\mathrm{CC}$ bond for the $\mathrm{V}$ and $\mathrm{Z}$ states of $90^{\circ}$-twisted ethene as a function of a) pyramidalization and b) scissoring. 


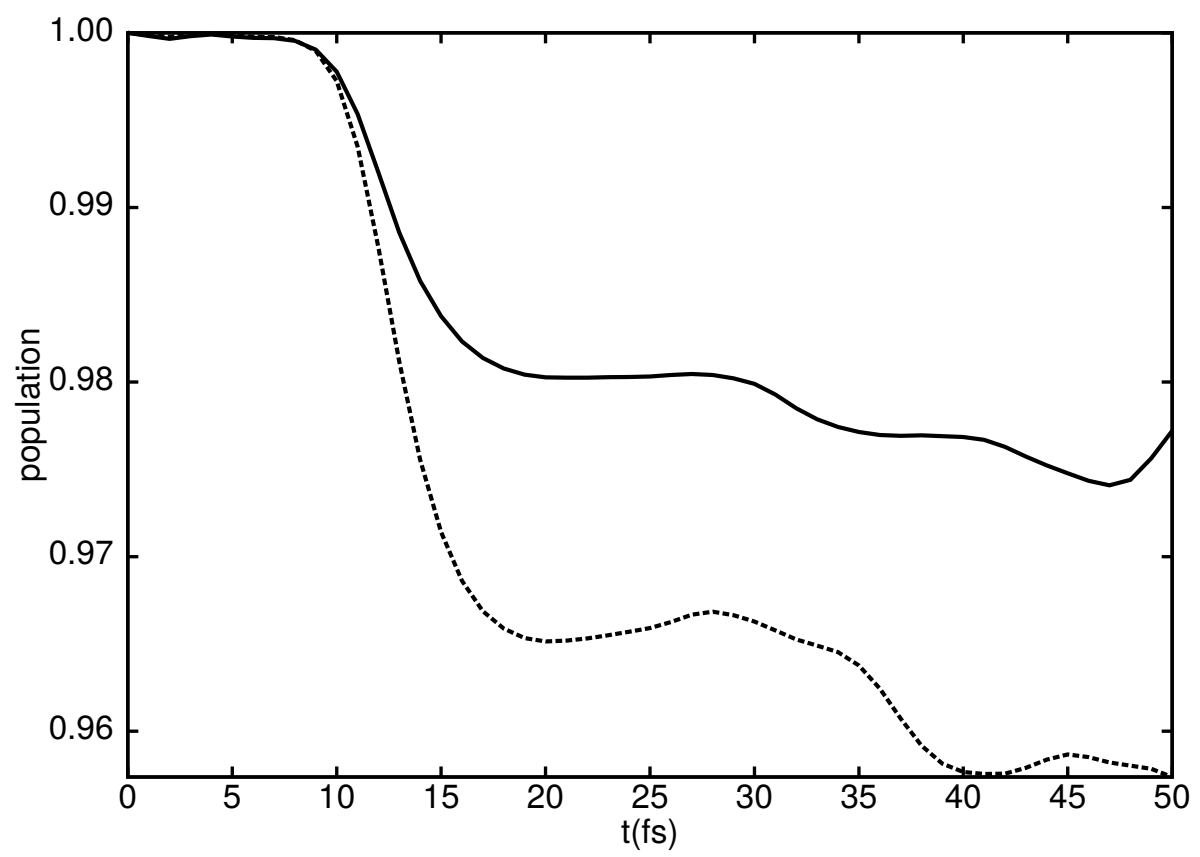

Figure 2: Time evolution of the population probability of the $\mathrm{V}$ state for a $\varphi, \vartheta$ (full line) and a $\varphi, \alpha$ (dotted line) wave-packet propagation, respectively. 